\title{
Temperature adaptation in yeasts: the role of fatty acids
}

\author{
M. SUUTARI, ${ }^{*}$ K. LiUKKonen and S. LaAKSo \\ Helsinki University of Technology, Department of Chemical Engineering, Laboratory of Biochemistry and Microbiology, \\ SF-02150 Espoo, Finland
}

(Received 2 January 1990; revised 12 March 1990; accepted 2 April 1990)

\begin{abstract}
Studies on the yeasts Candida oleophila, Candida utilis, Lipomyces starkeyi, Rhodosporidium toruloides and Saccharomyces cerevisiae revealed the existence of three different temperature adaptation responses involving changes in fatty acid composition. These conclusions were drawn by determining the growth rates, total cellular fatty acid content, fatty acid composition, degree of unsaturation, and the mean chain length of fatty acids over a range of growth temperatures. Within temperatures permitting growth, there were no changes in the major fatty acids of any of the yeasts, but the absolute amounts and relative compositions of the fatty acids did alter. In $S$. cerevisiae there were temperature-induced changes in the mean fatty acid chain length, whereas in $R$. toruloides there were changes in the degree of unsaturation. $C$. oleophila, $C$. utilis and $L$. starkeyi showed both responses, depending on whether the growth temperature was above or below $20-26{ }^{\circ} \mathrm{C}$. Below $20-26{ }^{\circ} \mathrm{C}$ temperaturedependent changes were observed in the mean chain length whereas above $20-26^{\circ} \mathrm{C}$ there were changes in the degree of unsaturation.
\end{abstract}

\section{Introduction}

Fatty acids play an important role in determining the physicochemical properties of membrane lipids. Therefore micro-organisms have different species-specific fatty acids (McElhaney, 1976). Analysis of these characteristic fatty acids can sometimes be used for strain identification purposes (Lechevalier \& Lechevalier, 1989). However, the relative amounts of the fatty acids may be altered by changes in external conditions such as temperature (Cronan, 1978). Less is known as to whether these temperature-induced changes are essential for survival and whether the capability of an organism to adapt to extreme temperature really reflects its predetermined ability to alter the relative fatty acid composition of membrane lipids (Neidleman, 1987). The mechanisms of temperature adaptation probably involve many cellular functions and components, but membrane fluidity and its maintenance by changes in fatty acid composition can hardly be ignored. How the fatty acid changes are related to the regulation of membrane-bound enzymes has been reviewed by Brenner (1984), Cronan \& Gelmann (1975) and Sandermann (1978).

More extensive information on microbial temperature adaptation mechanisms would also be valuable in many modern areas of biotechnology ranging from medical microbiology and immunology to food hygiene, pro- cessing and fermentation. In addition, interest in microbes as producers of speciality lipids is rapidly increasing (Ratledge \& Boulton, 1985): These considerations led us to study the temperature-dependent changes of fatty acids in several different types of yeast. The yeasts contain a type $\mathrm{I}$ fatty acid synthetase and $\mathrm{O}_{2-}$ dependent desaturases (Fulco, 1974; Bloch \& Vance, 1977; Wakil et al., 1983), and when grown in the presence of excess $\mathrm{O}_{2}$ could offer a wide spectrum of changes in membrane fatty acids. Measurements of fatty acid composition were made over a wide temperature range in order to examine the full extent of thermal dependence. The gathering of systematic data allows results to be presented which not only reveal the individuality of yeasts as modifiers of their fatty acid profiles but also general responses which can be related to temperature adaptation.

\section{Methods}

Strains and growth conditions. The yeasts used were Candida oleophila VTT-C-78088 (VTT, Technical Research Centre of Finland), Candida utilis VTT-C-84147, Rhodosporidium toruloides VTT-C-82132, Saccharomyces cerevisiae B I Lh 306 and Lipomyces starkeyi DSM 70295 (DSM, German Collection of Micro-organisms and Cell Cultures). The strains were stored in $10 \%(\mathrm{v} / \mathrm{v})$ glycerol at $-60^{\circ} \mathrm{C}$ and grown in $250 \mathrm{ml}$ 
Erlenmeyer flasks containing $50 \mathrm{ml}$ wort broth base. The flasks were shaken at 240 r.p.m. on a Gallenkamp orbital shaker/incubator (model INR $200010 \mathrm{~V}$ ). For preparation of inocula cells were grown to late exponential growth phase (150-200 Klett units); $1 \mathrm{ml}$ of this culture was transferred into a shake flask containing the same medium and allowed to grow to the exponential phase. This culture $(1 \mathrm{ml})$ was inoculated into four parallel flasks to be used for analyses. All these stages were carried out at each temperature studied. Growth was monitored with a Klett-Summerson colorimeter (filter no. 66). The fatty acid composition was determined in early exponential (50 Klett units), exponential ( $100 \mathrm{Klett}$ units), late exponential ( $150 \mathrm{Klett}$ units) and stationary ( $>200 \mathrm{Klett}$ units) phases. The total fatty acid content of cells was determined in the exponential phase.

To determine growth temperature minima and maxima the yeasts were grown on agar in individual cuvettes in a plate-type temperature gradient incubator (Gradiplate). The medium used was wort broth base containing $0.25 \%(\mathrm{v} / \mathrm{v})$ glycerol solidified with $2 \%(\mathrm{w} / \mathrm{v})$ agar.

Analyses. To determine the fatty acid composition samples $(5 \mathrm{ml})$ of culture suspensions were centrifuged for $10 \mathrm{~min}(5000 \mathrm{~g})$. The supernatant was removed and the cells were resuspended in $5 \mathrm{ml}$ of tapwater. Centrifugation was repeated, the water was removed and cells were frozen under a stream of $\mathrm{N}_{2}$ and stored $\left(-20^{\circ} \mathrm{C}\right)$ for $1-10 \mathrm{~d}$. Cells were suspended in an excess $(1 \mathrm{ml})$ of saponification reagent containing $3.7 \mathrm{M}-\mathrm{NaOH}$ in $49 \%(\mathrm{v} / \mathrm{v})$ methanol. Samples were flushed with $\mathrm{N}_{2}$, mixed, kept at $100^{\circ} \mathrm{C}$ in closed tubes for $5 \mathrm{~min}$, mixed and returned to a $100^{\circ} \mathrm{C}$ water-bath for $25 \mathrm{~min}$. The sample was cooled down to room temperature and $4 \mathrm{ml}$ of methylation reagent was added. The methylation reagent was $3.3 \mathrm{M}-\mathrm{HCl}$ in $48 \%$ (v/v) methanol. The sample was mixed, held at $80^{\circ} \mathrm{C}$ in a water-bath for $10 \mathrm{~min}$ and cooled down to room temperature. Fatty acid methyl esters were extracted in $1.5 \mathrm{ml}$ hexane/methyl tert-butyl ether solution $(1: 1, \mathrm{v} / \mathrm{v})$. The sample was shaken vigorously for $10 \mathrm{~min}$ and the lower phase was removed with a Pasteur pipette. The sample was washed by shaking for $5 \mathrm{~min}$ with $3 \mathrm{ml}$ $0.3 \mathrm{M}-\mathrm{NaOH}$. The sample was centrifuged $(5000 \mathrm{~g})$ for $20 \mathrm{~min}$ and the upper phase was removed. The sample was then dried with anhydrous $\mathrm{Na}_{2} \mathrm{SO}_{4}$, flushed with nitrogen and analysed by GLC. To determine the cellular lipid content the rest of the yeast culture suspension was harvested and washed with water as described above. The yeast cells were lyophilized and weighed, an internal standard (heptadecanoic acid methyl ester, Sigma) was added and the fatty acid composition was determined as described above. The major fatty acids were identified from their GLC peak retention times relative to fatty acid methyl ester standards (Sigma). GLC was done using a Hewlett-Packard model $5890 \mathrm{~A}$ gas chromatograph equipped with a flame ionization detector, a capillary inlet system and a model $7673 \mathrm{~A}$ high-speed automatic liquid sampler with a $10 \mu \mathrm{l}$ syringe. The GLC conditions were as follows: HPFFAP $(25 \mathrm{~m} \times 0.2 \mathrm{~mm} \times 0.3 \mu \mathrm{m})$ column; carrier gas helium, column flow-rate approx. $1.0 \mathrm{ml} \mathrm{min}^{-1}$; total hydrogen flow-rate to the detector $40 \mathrm{ml} \mathrm{min}{ }^{-1}$; make-up gas, helium, flow-rate $30 \mathrm{ml} \mathrm{min}^{-1}$; septum purge flow-rate $1-2 \mathrm{ml} \mathrm{min}^{-1}$; split ratio $1: 20$; column inlet pressure $150 \mathrm{kPa}$; injector temperature $250^{\circ} \mathrm{C}$; detector temperature $250^{\circ} \mathrm{C}$; oven temperature was programmed from $70^{\circ} \mathrm{C}$ to $200^{\circ} \mathrm{C}$ at the rate of $25^{\circ} \mathrm{C} \mathrm{min}-1$. Peak areas were measured using a Hewlett-Packard model 3396A integrator.

Calculations. The growth rate was determined from the slope of the growth curve in the exponential growth phase. The relative fatty acid composition was estimated as a percentage of the total peak area and expressed as a mean value from early exponential and exponential growth phases. The absolute amount of the individual fatty acids was calculated as a percentage of the cell dry weight. The total fatty acid content of the cells was defined as the sum of cellular fatty acid methyl esters. The degree of unsaturation $\left(\Delta \mathrm{mol}^{-1}\right)$ in the lipid fraction was calculated as $\Delta \mathrm{mol}^{-1}=[1.0(\%$ monoene $)+2.0(\%$ diene $)+3.0$
$(\%$ triene) $] / 100$. The mean fatty acid chain length was expressed as the ratio of $\mathrm{C}_{16} / \mathrm{C}_{18}$ acids. The standard deviation $(n=8)$ was $<0.9 \%$.

Chemicals. Components of growth media and chemicals were obtained from Merck, unless otherwise stated.

\section{Results}

\section{Effect of temperature on growth}

The temperatures used in shake flask cultivations appeared to cover the temperature range permitting growth of the yeasts (Table 1). The minimum growth temperature used $\left(10^{\circ} \mathrm{C}\right)$ exceeded the actual minima by 3.8-10 centigrade degrees, as determined by the Gradiplate technique. Similarly, the highest temperatures were less than 4 centigrade degrees below the maxima. Each of the yeasts grew over a temperature range $(\Delta T)$ of $27.5-$ 36. 0 centigrade degrees.

Fig. 1 illustrates the growth rates as a function of temperature. Below $25^{\circ} \mathrm{C}$ the correlations were linear and positive. At higher temperatures the rates declined in a pronounced fashion. The fatty acid compositions during both the increasing and the declining phases were investigated.

Table 1. Correlation of shake-flask temperatures with the real growth temperature ranges as determined by the Gradiplate technique

\begin{tabular}{|c|c|c|c|c|}
\hline \multirow[b]{2}{*}{ Yeast } & \multirow{2}{*}{$\begin{array}{c}\text { Temperatures in } \\
\text { shake-flask } \\
\text { experiments } \\
\left({ }^{\circ} \mathrm{C}\right)\end{array}$} & \multicolumn{3}{|c|}{$\begin{array}{c}\text { Gradiplate } \\
\text { determinations }\end{array}$} \\
\hline & & $\begin{array}{l}T_{\min } \\
\left({ }^{\circ} \mathrm{C}\right)\end{array}$ & $\begin{array}{l}T_{\max } \\
\left({ }^{\circ} \mathrm{C}\right)\end{array}$ & $\begin{array}{l}\Delta T \\
\left({ }^{\circ} \mathrm{C}\right)\end{array}$ \\
\hline C. utilis & $10,12,15,20,26,30,35,40$ & $6 \cdot 3$ & $39 \cdot 7$ & $33 \cdot 4$ \\
\hline C. oleophila & $10,12,15,20,26,30$ & $<0$ & $34 \cdot 3$ & $>34 \cdot 3$ \\
\hline L. starkeyi & $10,15,20,26,30$ & $5 \cdot 4$ & $33 \cdot 1$ & $27 \cdot 7$ \\
\hline R. toruloides & $10,15,20,26,30$ & $<0$ & 33.9 & $>33.9$ \\
\hline S. cerevisiae & $10,12,15,20,25,30,35$ & 2.8 & $39 \cdot 3$ & $36 \cdot 5$ \\
\hline
\end{tabular}



Fig. 1. Growth rates as a function of culture temperature. $\triangle, C$. oleophila; , C. utilis; $\Delta$, L. starkeyi; $\square, R$. toruloides; $\square, S$. cerevisiae. 

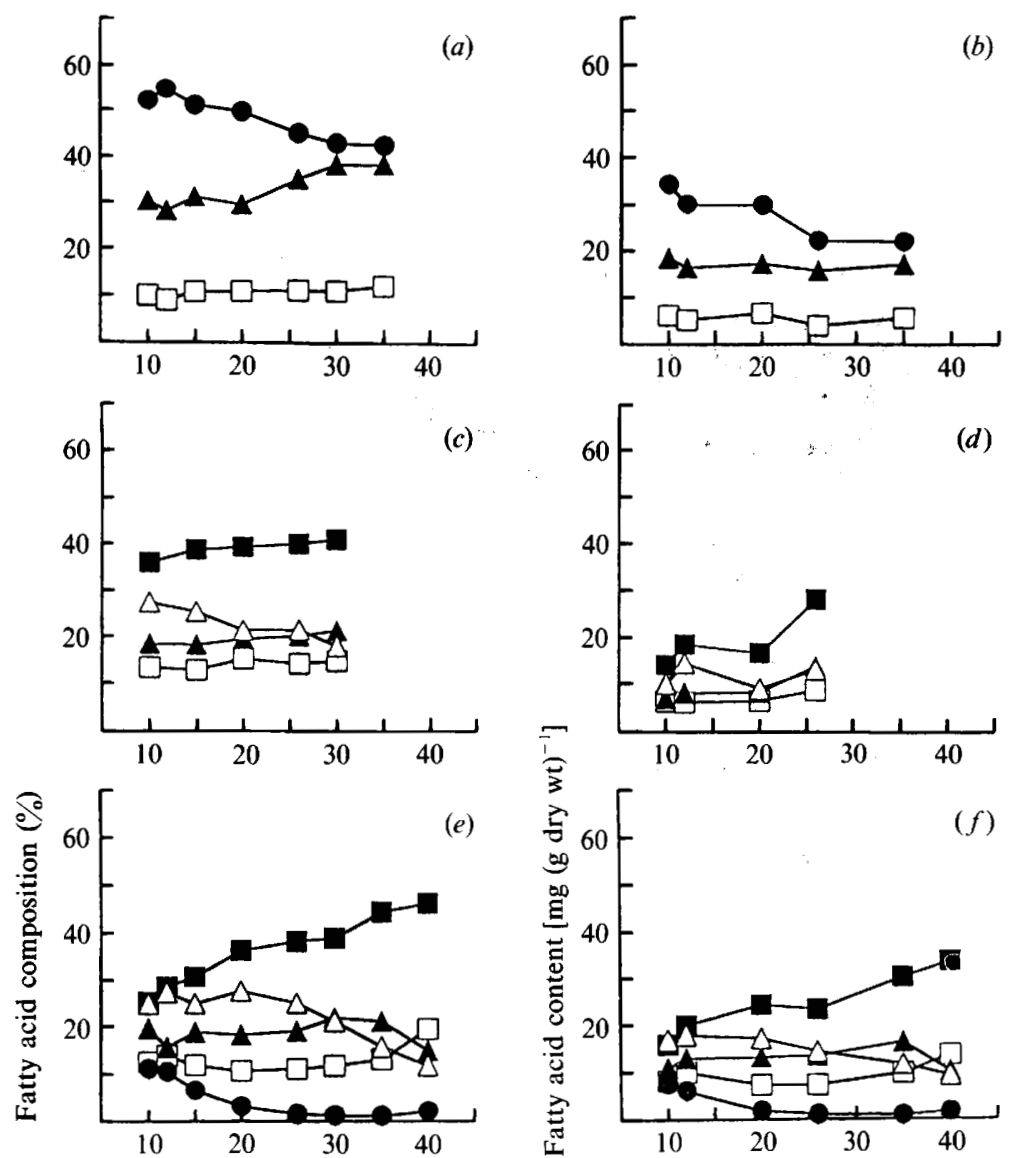

$(e)$
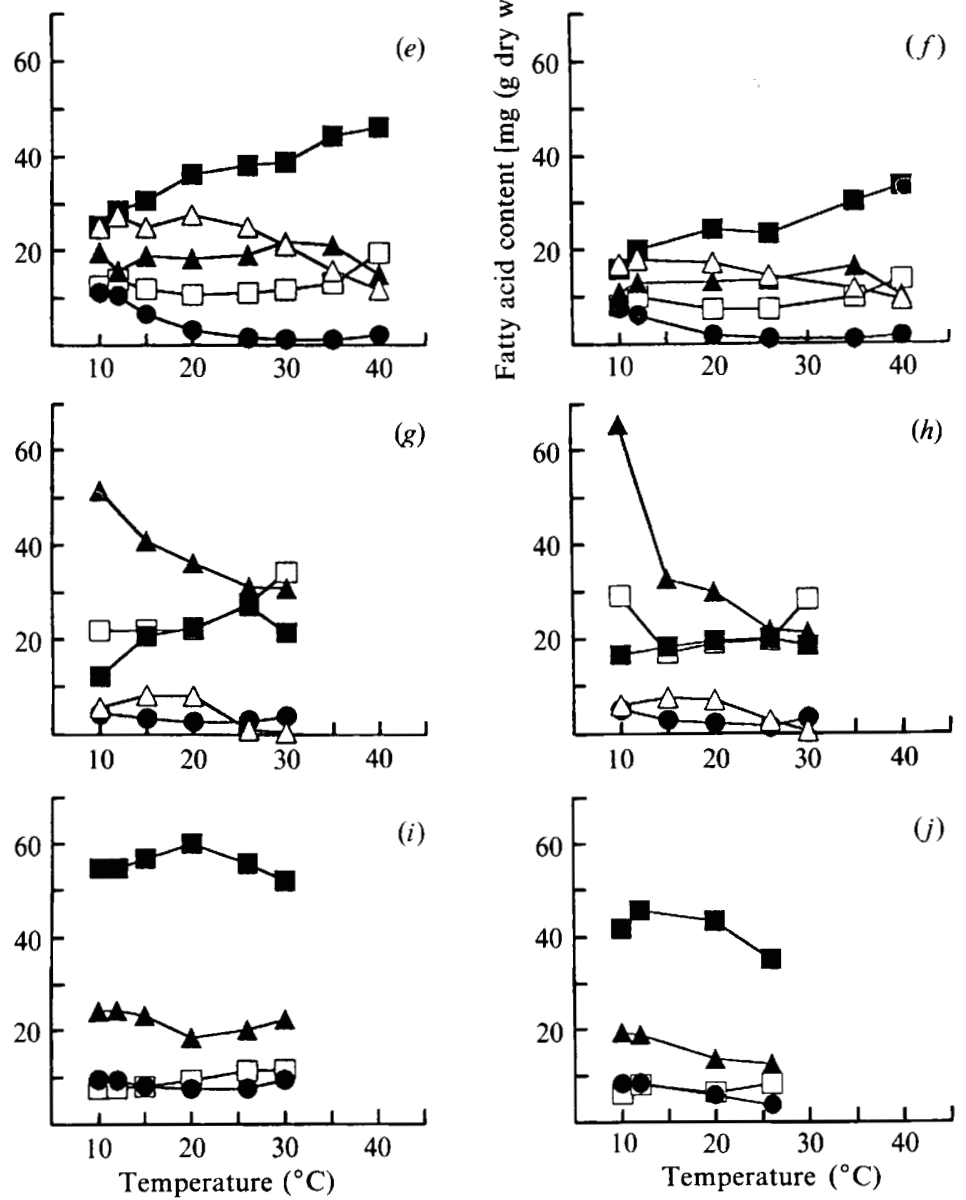

Fig. 2. Temperature-induced variations in the fatty acid profiles expressed as relative $(a, c, e, g, i)$ and absolute $(b, d, f, h, j)$ contents. $(a$, b) S. cerevisiae; $(c, d)$ R. toruloides; $(e, f)$ C. utilis; $(g, h)$ L. starkeyi; $(i, j)$ C. oleophila. $\square$, Palmitic acid;, palmitoleic acid; $\boldsymbol{\Delta}$, oleic acid; $\square$, linoleic acid; $\triangle$, linolenic acid. 


\section{Effect of growth phase on fatty acid composition}

The yeasts contained three to five characteristic fatty acids which accounted for more than $90 \%$ of the total amount of fatty acids (Fig. 2). The major fatty acids were palmitic $(16: 0)$, palmitoleic $(16: 1)$, oleic $(18: 1)$, linoleic $(18: 2)$ and linolenic acid $(18: 3)$. The relative compositions of major fatty acids changed less than $10 \%$ with the growth phase except in $L$. starkeyi. The ratio of $\mathrm{C}_{16} / \mathrm{C}_{18}$ fatty acids did not change, except in $L$. starkeyi, in which the proportion of $\mathrm{C}_{16}$ acids increased. The degree of unsaturation also changed only in L. starkeyi, in which it decreased from the early exponential to the late stationary phase. This can be explained by the ability of L. starkeyi to accumulate palmitic and oleic acids in favour of palmitic acid upon cessation of growth (Suzuki \& Hasegawa, 1974). Minor fatty acids were detected in all the yeasts ( $<2.8 \%$ of the total), but they showed little growth-phase- or temperature-dependent changes.

Effect of growth temperature on the content of palmitic and palmitoleic acids

Palmitic acid was the only major saturated fatty acid present in all the strains studied and its content varied only slightly with the growth temperature (Fig. 2). Its amount, expressed as relative content varied most in $C$. oleophila $(4.0 \%)$; when expressed with respect to cell dry weight it varied most in $R$. toruloides $(2.7 \%)$. There was a general trend towards an increased relative amount of palmitic acid with increasing growth temperature. There were, however, two exceptions. The palmitic acid content in C. utilis showed a definite increase at $40^{\circ} \mathrm{C}$, i.e. near the maximum growth temperature, while the content of oleic acid decreased concomitantly. The lipidaccumulating yeast, "L. starkeyi, showed a tendency to have a lower relative palmitic acid content with decreasing temperature. However, below $15^{\circ} \mathrm{C}$ an increase in its absolute amount was found.

The absolute palmitoleic acid content increased with decreasing temperature in $C$. oleophila, $C$. utilis, $L$. starkeyi and $S$. cerevisiae. In relative, but not in absolute terms the palmitoleic acid also increased somewhat near the upper temperature limit of C. oleophila, C. utilis and $L$. starkeyi. In $R$. toruloides palmitoleic acid was only present in amounts less than $0.6 \%$.

Effect of growth temperature on the content of oleic, linoleic and linolenic acids

Oleic acid was the only unsaturated fatty acid present in all the strains investigated (Fig. 2). Temperature-induced variations in the proportion of oleic acid were speciesspecific. In $C$. oleophila, $C$. utilis and $R$. toruloides oleic acid content varied maximally by $10 \%$ and $7 \%$ in relative and absolute terms, respectively. In $S$. cerevisiae, which contained only three major fatty acids, the relative oleic acid content varied between $28 \cdot 1-38 \cdot 6 \%$. However, the absolute amounts varied by only $2.5 \%$ (between 15.9 $18.4 \%$ ). In $L$. starkeyi the relative and absolute amounts of oleic acid increased with decreasing temperature.
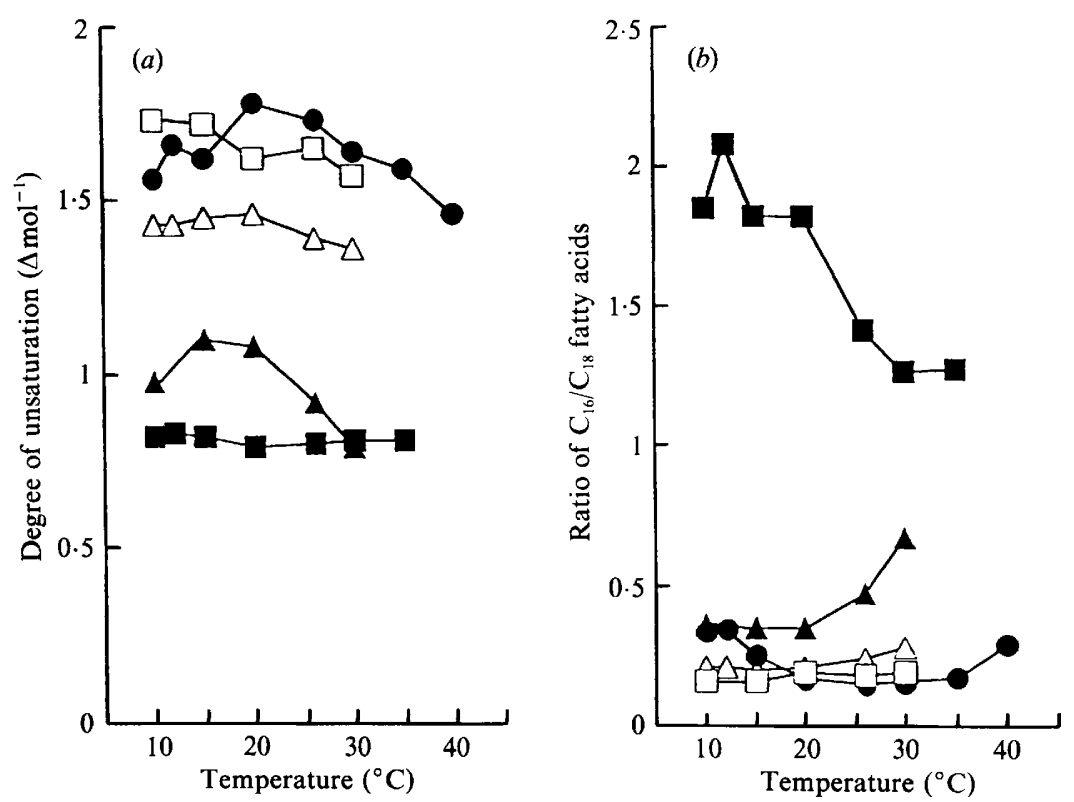

Fig. 3. Changes in the degree of unsaturation $(a)$ and the ratio of $C_{16} / C_{18}$ fatty acids $(b)$ as a function of growth temperature in the exponential growth phase. $\triangle$, C. oleophila; , C. utilis; $\Delta$, L. starkeyi; $\square, R$. toruloides; $\square, S$. cerevisiae. 


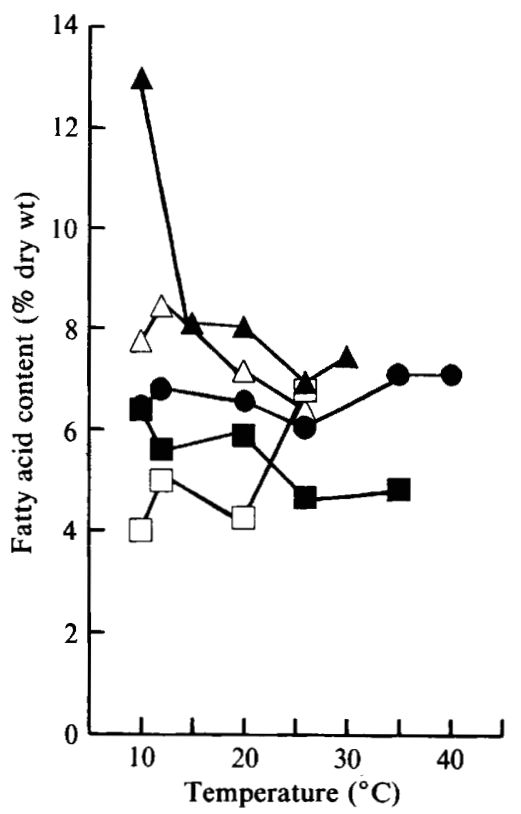

Fig. 4. Cellular fatty acid content at different growth temperatures in exponentially growing yeast cultures. $\triangle, C$. oleophila;,$C$. utilis; $\mathbf{\Delta}$, L. starkeyi; $\square, R$. toruloides; $\square, S$. cerevisiae.

The relative amount of linoleic acid in C. utilis and $R$. toruloides increased almost linearly with the growth temperature. $L$. starkeyi behaved similarly with the exception of the highest temperature (around $30^{\circ} \mathrm{C}$ ). In C. oleophila a similar positive correlation was also seen up to $20^{\circ} \mathrm{C}$. At higher temperatures (between $20-30{ }^{\circ} \mathrm{C}$ ) the correlation became negative. Linoleic acid was not detected in $S$. cerevisiae.

The relative and absolute amounts of linolenic acid in $C$. utilis and $L$. starkeyi were constant at low growth temperatures (up to $20^{\circ} \mathrm{C}$ ) but decreased with further increases in temperature. In $C$. utilis these reductions were accompanied by a concomitant increase in linoleic acid. - In $R$. toruloides linolenic acid was apparently replaced by linoleic acid and to a lesser extent by oleic acid with increasing temperature when expressed in terms of relative fatty acid proportions.

$S$. cerevisiae and C. oleophila did not contain trienoic acids over the whole temperature range studied.

\section{Effect of temperature on the fatty acid content, and the degree of fatty acid unsaturation and mean chain length}

In $S$. cerevisiae (Fig. $3 a$ ) the degree of unsaturation did not vary as a function of the temperature of the growth medium. The fatty acid content of this yeast (Fig. 4) and the $\mathrm{C}_{16} / \mathrm{C}_{18}$ ratio (Fig. $3 b$ ) increased with decreasing temperature. In $R$. toruloides changes in the degree of unsaturation were significant. This value changed from

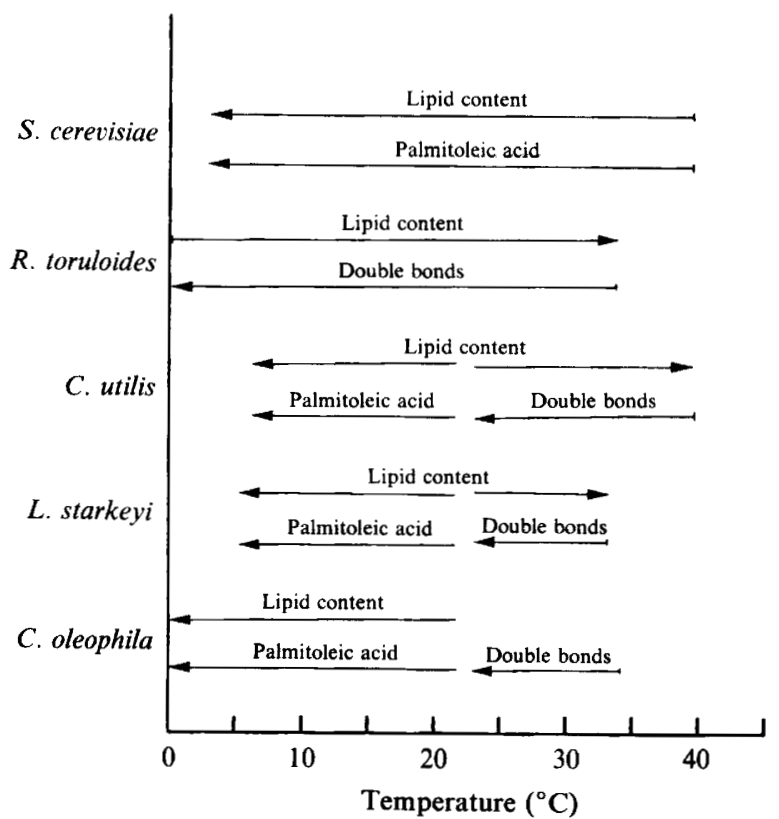

Fig. 5. Summary scheme of the lipid-related responses involved in temperature adaptation of different yeasts.

1.57 to 1.73 with decreasing temperature while the fatty acid content decreased. The mean fatty acid chain length in this organism was constant over the temperature range studied. In C. utilis and L. starkeyi the dependence of the fatty acid content and the degree of unsaturation on temperature showed biphasic behaviour with a minimum and maximum at $20-26^{\circ} \mathrm{C}$, respectively. Both above and below this temperature range the fatty acid content increased. $C$. oleophila behaved similarly to $C$. utilis and $L$. starkeyi below $26^{\circ} \mathrm{C}$. The trends of changes in the degree of unsaturation were the opposite to those found for fatty acid content. Fig. 3(b) shows that in $C$. oleophila, C. utilis and $L$. starkeyi the ratio of $\mathrm{C}_{16} / \mathrm{C}_{18}$ fatty acids increased with increasing temperature above $20^{\circ} \mathrm{C}$. Below $20^{\circ} \mathrm{C}$ an opposite correlation was seen in $C$. utilis.

\section{Discussion}

This study identified three responses to temperature of the fatty acid composition of yeasts (Fig. 5).

The first response was seen in $S$. cerevisiae, which reacted to temperature changes by regulating the palmitoleic acid content. As a result the mean chain length of fatty acids (Fig. $3 b$ ) changed with temperature as demonstrated also by Okuyama et al. (1979). The degree of unsaturation, often proposed to vary with temperature in S. cerevisiae, was virtually unaltered. This had also been suggested by Hunter \& Rose (1972). 
The second response was seen in $R$. toruloides, which reacted to increasing temperature by lowering the degree of fatty acid unsaturation (Fig. $3 a$ ) and increasing the fatty acid content (Fig. 4). Regulation by alteration of the mean chain length can be ruled out, because the level of palmitic acid remained unchanged and the palmitoleic acid content was low over the whole temperature range. High temperatures might favour lipid accumulation in $R$. toruloides.

C. utilis demonstrated a third type of adaptation because, depending on the temperature range, it could switch from one response to another. Below $20-26^{\circ} \mathrm{C}$ the ratio of $\mathrm{C}_{16} / \mathrm{C}_{18}$ fatty acids and the fatty acid content decreased with increasing temperature, analogous to the response seen in $S$. cerevisiae. According to Davis et al. (1981) and Coolbear et al. (1983) a shortening of the fatty acid chain length and insertion of the first double bond has a much greater effect on lipid fluidity than does insertion of subsequent double bond(s). This might explain the increasing monoenoic acid content as the temperature is lowered. McMurrough \& Rose (1971) have also suggested that the maintenance of high proportions of polyunsaturated fatty acids is not a prerequisite for the growth of $C$. utilis at suboptimal temperatures. However, above $20-26^{\circ} \mathrm{C}$ the degree of unsaturation decreased and the fatty acid content increased with temperature similarly to the response of $R$. toruloides.

L. starkeyi showed a biphasic temperature adaptation behaviour similar to that of $C$. utilis. In addition, the fatty acid content (Fig. 4) increased substantially below $20^{\circ} \mathrm{C}$ at the expense of palmitic and oleic acids. In C. oleophila the absolute monoenoic acid content and fatty acid content (Fig 4) decreased with increasing temperature. However, above $20^{\circ} \mathrm{C}$ only the degree of unsaturation (Fig. $3 a$ ) decreased significantly.

This study revealed a much wider range of adaptive responses than previously expected. The temperature range from $20-26{ }^{\circ} \mathrm{C}$ appeared to be critical in determining the mode of response in those yeasts showing a biphasic behaviour, but this cannot be explained from the present data. Neither the growth rate maxima nor dissolved $\mathrm{O}_{2}$ concentration coincided with this temperature range. A deeper understanding of the mechanisms involved in these responses requires further information on fatty acid changes at the level of membrane phospholipids and/or stored triacylglycerols. This work is in progress in our laboratory.

We thank Mrs U. Åmman for her technical assistance. The financial support of the Technology Development Centre (TEKES) is gratefully acknowledged.

\section{References}

BLOCH, K. \& VANCE, D. (1977). Control mechanisms in the synthesis of saturated fatty acids. Annual Review of Biochemistry 46, 263-298.

BRENNER, R. R. (1984). Effect of unsaturated acids on membrane structure and enzyme kinetics. Progress in Lipid Research 23, 69-96.

Coolbear, K. P., Berde, C. B. \& KeOugh, K. M. W. (1983). Gel to liquid-crystalline phase transitions of aqueous dispersions of polyunsaturated mixed-acid phosphatidylcholines. Biochemistry 22, 1466-1473.

Cronan, J. E., JR (1978). Molecular biology of bacterial membrane lipids. Annual Review of Biochemistry 47, 163-189.

Cronan, J, E. \& Gelmann, E. P. (1975). Physical properties of membrane lipids: biological relevance and regulation. Bacteriological Reviews 39, 232-256.

Davis, P. J., Fleming, B. D., Coolbear, K. P. \& Keough, K. M. W. (1981). Gel to liquid-crystalline transition temperatures of water dispersions of two pairs of positional isomers of unsaturated mixedacid phosphatidylcholines. Biochemistry 20, 3633-3636.

Fulco, A. J. (1974). Metabolic alterations of fatty acids. Annual Review of Biochemistry 43, 215-241.

HUNTER, K. \& ROSE, A. H. (1972). Lipid composition of Saccharomyces cerevisiae as influenced by growth temperature. Biochimica et Biophysica Acta 260, 639-653.

LeCHEVAliER, H. \& LeChEVAlier, M. P. (1989). Chemotaxonomic use of lipids - an overview. In Microbial Lipids, pp. 869-902. Edited by C. Ratledge \& S. G. Wilkinson. London: Academic Press.

MCELHANEY, R. N. (1976). The biological significance of alterations in the fatty acid composition of microbial membrane lipids in response to changes in environmental temperature. In Extreme Environments Mechanisms of Microbial Adaptation. Edited by M. R. Heinrich. New York: Academic Press.

McMurrough, I. \& Rose, A. H. (1971). Effects of temperature variation on the fatty acid composition of Candida utilis. Journal of Bacteriology 107, 753-758.

NeIDLEMAN, S. L. (1987). Effects of temperature on lipid unsaturation. Biotechnology and Genetic Engineering Reviews 5, 245-268.

Oxuyama, H., Saito, M., Joshi, V. C., Gunsberg, S. \& Wakil, S. J. (1979). Regulation by temperature of the chain length of fatty acids in yeast. Journal of Biological Chemistry 254, 12281-12284.

Ratledge, C. \& Boulton, C. A. (1985). Fats and oils. In Comprehensive Biotechnology, vol. 3, pp. 983-1003. Edited by H. W. Blanch, S. Drew \& D. I. C. Wang. Oxford: Pergamon Press.

SANDERmanN, H., JR (1978). Regulation of membrane enzymes by lipids. Biochimica et Biophysica Acta 515, 209-237.

SUZUKI, T. \& HASEGAWA, K. (1974). Lipid molecular species of Lipomyces starkeyi. Agricultural and Biological Chemistry 38, 1371-1476.

WAKIL, S. J., Stoops, J. K. \& JoshI, V. C. (1983). Fatty acid synthesis and its regulation. Annual Review of Biochemistry 52, 537-579. 